\title{
2.4 GHz L-band passively harmonic mode locked Er-doped fiber laser based on carbon nanotubes film
}

\author{
Qianqian Huang, Zinan Huang, Mohammed Al Araimi, Aleksey Rozhin, and Chengbo Mou
}

\begin{abstract}
We experimentally demonstrate a passively harmonic mode locked (PHML) Er-doped fiber laser with pump power efficiency up to $17 \mathrm{MHz} / \mathrm{mW}$ operating at L-band based on single walled carbon nanotubes polyvinyl alcohol (SWCNTs-PVA) film. Under $233 \mathrm{~mW}$ pump power, the stable pulse train at 1594.97 $\mathrm{nm}$ with $40.5 \mathrm{~dB}$ side mode suppression ratio (SMSR) and $742 \mathrm{fs}$ pulse duration is obtained at a repetition rate of $1.923 \mathrm{GHz}$, corresponding to $170^{\text {th }}$ harmonic of the fundamental frequency. Under optimized intracavity conditions, the pulses frequency is able to scale up to $2.415 \mathrm{GHz}$ with a high level of $40 \mathrm{~dB}$ SMSR, which to the best of our knowledge, is the highest value yet reported from a L-band PHML fiber laser incorporating SWCNTs as saturable absorber (SA). Such high repetition rate and stable fiber laser operating at $L$ band may be desirable for various applications.
\end{abstract}

Index Terms-mode locked fiber lasers, mode locking, nanomaterials

\section{INTRODUCTION}

$\mathrm{P}$ RESENT research activities on ultrashort pulse fiber lasers with up to $\mathrm{GHz}$ range repetition rate have been extensively explored on account of their potential applications in optical frequency metrology [1], high speed optical sampling [2] and modern optical communication system [3]. Especially, for the modern optical communication system, the conventional Cband telecommunication window has been incapable of supplying the demand of persistent increasing of communication capacity. For addressing the problem, extending optical telecommunication region to $\mathrm{L}$ band is a key issue. Therefore, there is no doubt that investigating L-band fiber laser with high repetition rate via PHML method is of great importance.

It is already widely acknowledged that active mode locking [4], short cavity [5] as well as PHML [6] have been considered as effective methods to achieve high repetition rate pulses. In a PHML laser, when high pump light launches into the laser

\footnotetext{
Manuscript received XXXXXX. We would like to express our gratitude to the National Natural Science Foundation of China (NSFC) $(61605107,61975107)$ and the open fund (Grant No. IPOC2017B010) of the Institute of Information Photonics and Optical Communications at BUPT, China. C. Mou acknowledges Young Eastern Scholar Program at Shanghai Institutions of Higher Learning (QD2015027), "Young 1000 Talent Plan" Program of China and RAEng and the Leverhulme Trust Senior Research Fellowships (LTSRF1617/13/57). This paper was partially revised in the baby delivery room. C. Mou would like to acknowledge his wife for gifting his first son. C.Mou would also like to welcome mini-Mou, Hello World.

Q. Huang, Z. Huang and C. Mou are with The Key Lab of Specialty Fiber Optics and Optical Access Network, Shanghai Institute for Advanced Copyright (c) 2019 IEEE
}

cavity, the single pulse circulating in the cavity would split into several pulses caused by peak power limiting and energy quantization effects. These multiple pulses do not distribute uniformly for general case. However, PHML occurs when the split pulses in a round-trip arrange themselves automatically exhibiting equal temporal spacing and uniform amplitude under appropriate conditions. At this time, the repetition rates of the laser then scale up some multiple of the cavity length defined fundamental repetition rate. Consequently, without an extra modulator or realizing impractical cavity length, a PHML fiber laser shows the superiority for the achievement of high repetition rate ultrashort pulses compared with other methods mentioned above.

Since Grudinin et al firstly demonstrated the generation of PHML using nonlinear polarization rotation (NPR) [7], NPR has been utilized intensively and thanks to the advantages of sub-picosecond recovery time and wavelength-independent behavior. However, the NPR technique processes poor environmental stability. Fortunately, the physical SAs including semiconductor saturable absorber mirror (SESAM) [8], CNTs [9]-[11] and other types of nanomaterials [6] can remove such limitation and meanwhile show self-starting property in PHML. Nevertheless, SESAM exhibits some shortcomings of complex manufacture, narrow working bandwidth and high cost. Alternatively, CNTs have attracted much interest in HML generation recently, benefitting from the ultra-short recovery time, broadband operation range and environmental robustness. The first demonstration of HML based on CNTs was reported in 2010 resulting in repetition rates of $328.4 \mathrm{MHz}$ with $30 \mathrm{~dB}$ SMSR, showing relatively unstable operation state [9]. Mou et al achieved $245 \mathrm{MHz}$ repetition rate pulses with $40 \mathrm{~dB}$ SMSR, exhibiting much better stability [10]. However, the repetition rate is constrained to several hundreds of megahertz. In order to further explore the capability of PHML, Jun et al carefully optimized the cavity dispersion in an evanescent-type CNTs based fiber laser and

Communication and Data Science, Joint International Research Laboratory of Specialty Fiber Optics and Advanced Communication, Shanghai University, Shanghai, 200444 P. R. China (e-mail: cecilin@i.shu.edu.cn; zinanhuang@shu.edu.cn; corresponding author mouc1@shu.edu.cn).

M. AlAraimi is with Higher College of Technology, Al-Khuwair, PO BOX 74, Postal Code 133, Sultanate of Oman (email: mohammed.alaraimi@hct.edu.om)

A. Rozhin is with Aston Institute of Photonic Technologies (AIPT), Aston University, Birmingham, B4 7ET, United Kingdom (e-mail: a.rozhin@aston.ac.uk ).

Color versions of one or more of the figures in this paper are available online at http://ieeexplore.ieee.org

Digital Object Identifier Xxxxxxxxxxx 
realized $5 \mathrm{GHz}$ pulses at C-band successively [11]. Recently, our group has extended the laser operation band to $\mathrm{L}$ band and demonstrated that the pulse energy was ultimately conditional on the production of average dispersion and spectral bandwidth (DBP) while other cavity conditions were similar. In succession, $2.08 \mathrm{GHz}$ HML pulses were achieved [12].

In this letter, we propose a high-order PHML fiber laser operating at L-band based on SWCNTs-PVA film. When the pump power is set as $228 \mathrm{~mW}$, the pulse frequency varies from $1.8 \mathrm{GHz}$ to $2.415 \mathrm{GHz}$ under different polarization state. It is found that pulse duration sustains in the scale of 800 fs to 900 fs and SMSR is always more than $40 \mathrm{~dB}$. Compared to Ref [12], it shows dramatic improvements both in pulse duration and stability. The highest recorded repetition rate is $2.415 \mathrm{GHz}$, corresponding to $213^{\text {rd }}$ harmonic order. To the best of our knowledge, it is the highest repetition rate achieved thus far from L-band PHML lasers using CNTs. Also, it should be noted that our laser possesses pump power efficiency of 17.21 $\mathrm{MHz} / \mathrm{mW}$, which is much higher than previous reports [11,12]. Taking into account GHz L-band emission with good stability and femtosecond magnitude pulse duration, our laser may do contribution to some applications, especially, the modern optical communication system, spectroscopy etc.

\section{CHARACTERRISTICS OF CNTS-PVA FILM AND EXPERIMENTAL SET-UP}

\section{A. Characteristics of CNTS-PVA Film}
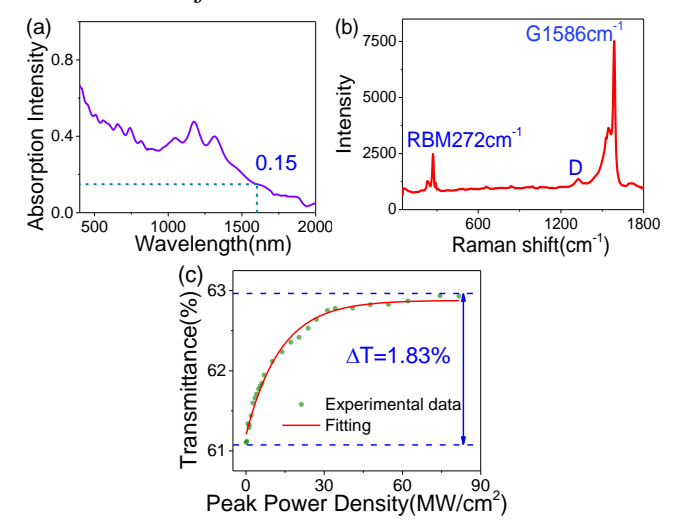

Fig. 1. The characteristics of the CNTs-PVA film (a) The linear absorption spectrum; (b) The Raman spectrum; (c) The measured nonlinear transmission.

The production process of SWCNTs-PVA film is described elsewhere [12].The absorption spectrum of the as-prepared SWCNTs-PVA film is depicted in Fig. 1(a). From Fig. 1(a), it is found that the absorption band is wide and the absorbance is nearly 0.15 at $1600 \mathrm{~nm}$, which gives rise to the possibility for laser emission at $\mathrm{L}$ band. Figure 1(b) plots the Raman spectrum measured by a $532 \mathrm{~nm}$ pump laser. The radial breathing mode (RBM) is about $272 \mathrm{~cm}^{-1}$, from which, we speculate that the resultant film exhibits both metallic and semiconducting properties and the mean diameter is reckoned to be $0.8 \mathrm{~nm}$. The existence of RBM makes a clear indication that our CNTs are single walled [13]. The nonlinear transmission of the CNTs film is measured with the assistance of a self-made mode locked fiber laser with central wavelength of $1597.34 \mathrm{~nm}$. As shown in Fig. 1(c), the measured modulation depth is $1.83 \%$, indicating that the SWCNTs-PVA film can realize mode locking at $\mathrm{L}$ band.

\section{B. Experimental set-up}

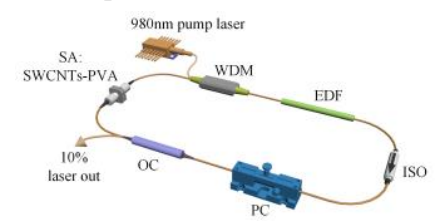

Fig. 2. L-band HML laser configuration based on SWCNTs-PVA film.

The L-band PHML fiber laser with SWCNTs-PVA film is schematically illustrated in Fig. 2. The laser is pumped by a 980 $\mathrm{nm}$ benchtop laser (OV LINK, Wuhan, China) which delivers up to a maximum power of $528 \mathrm{~mW}$ through a wavelengthdivision multiplexer (WDM). The light in cavity propagates clockwise with the assistant of polarization-independent isolator (PI-ISO). A polarization controller (PC) placed after PIISO is utilized to optimize the intracavity birefringence. The packaged SA is formed by sandwiching the SWCNTs-PVA film between two fiber connectors directly. The ring resonator is about $18.35 \mathrm{~m}$, comprising of $8.18 \mathrm{~m}$ Er-doped fiber (EDF Er30-4/125 from Liekki) acting as gain medium with absorption of $30 \mathrm{~dB} / \mathrm{m}$ at $1530 \mathrm{~nm}, 7.42 \mathrm{~m}$ single mode fiber (SMF) and $2.6 \mathrm{~m}$ OFS 980 fiber. Remarkably, the EDF length we use is much longer than most of the conventional lasers operating at C-band operation [14-17]. Particularly, the mode field diameter of the EDF is $6.5 \pm 0.5 \mu \mathrm{m}$, featuring slightly large nonlinear parameter than SMF. The group velocity dispersion (GVD) coefficients of EDF, SMF and OFS 980 are $+14.45 \mathrm{ps}^{2} / \mathrm{km},+4.5 \mathrm{ps}^{2} / \mathrm{km}$ and $-22.8 \mathrm{ps}^{2} / \mathrm{km}$, respectively. Therefore, the total net dispersion is $-0.039 \mathrm{ps}^{2}$ and the average dispersion is about $-2.14 \mathrm{ps}^{2} / \mathrm{km}$. It should be noted that the proposed laser is on purposely designed in the dispersionmanaged soliton regime with comparatively small net dispersion. On one hand, the DBP value at this point is relatively small, facilitating pulse splitting [12]. On the other hand, the generated pulse approximates stretched pulse, offering shorter pulse duration and better stability [5], [14].

From a 10:90 output coupler (OC), 10\% signal light is tapped out for pulse characterization through an $8 \mathrm{GHz}$ oscilloscope (OSC, KEYSIGHT DSO90804A) and a radio frequency (RF) spectrum analyzer (SIGLENT, SSA 3032X) with the help of a $12.5 \mathrm{GHz}$ photo-detector (PD, Newport 818- BB-51F). In addition, the pulse spectrum is ascertained by an optical spectrum analyzer (OSA, Yokogawa AQ6370C) while the pulse duration is detected by an autocorrelator (FEMTOCHROME, FR-103WS).

\section{RESUlTS AND DISCUSSION}

Only two operation states are observed with the presented laser, namely, multiple pulses with unceasing motion and PHML. When pump power increases to $80.3 \mathrm{~mW}$, mode locking occurs initially and multiple pulses in one round trip appear and move randomly at the same time. At this time, the pulses are at an undefined frequency and the corresponding optical spectrum is accompanied with clear continuous wave $(\mathrm{CW})$, indicating unstable state. Worthy of mentioning, single 

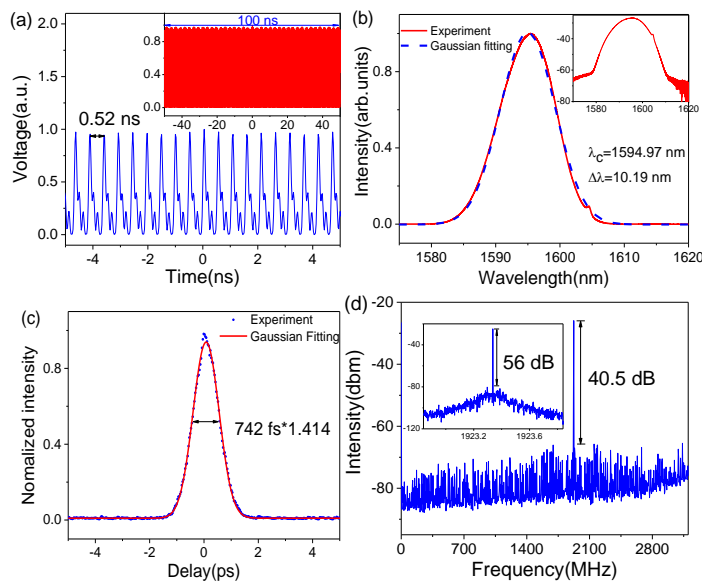

Fig. 3. The characteristics of $1.923 \mathrm{GHz}$ pulse train. (a) The recorded pulse train Inset: pulse train with the enlarged region of $100 \mathrm{~ns}$;(b) Linear optical spectrum with Gaussian shape assumption. Inset: the optical spectrum with $0.02 \mathrm{~nm}$ resolution; (c) The autocorrelation trace; (d) The RF spectra.

pulse state is never realized in the laser no matter how we adjust PC or pump power. It is believed that the long highly doped EDF with relatively small mode field diameter leads to slightly stronger nonlinear effect which may result in the unusual laser behavior. When higher pump power launches into the cavity, the pulses split further and continue to move around the cavity. At this point, HML can be realized in two cases. For the first case, the achievement of HML has to take several minutes or more when the unstable $\mathrm{CW}$ always coexists with the soliton. Meanwhile, it possesses poor stability as reflected in uneven pulse amplitude and low SMSR, showing similarity with that described elsewhere [15], [16]. Another solution is to rotate PC carefully until CW lasing disappears. As we do that, the pulses automatically organize themselves and the relatively steady HML is progressively formed within several seconds. Noted that the HML operations described in the following are always realized by this way.

Under $233 \mathrm{~mW}$ pump power, the $170^{\text {th }}$ harmonic at the repetition rate up to $1.923 \mathrm{GHz}$ was observed. Figure 3(a) plots the temporal waveforms, in which pulses are equidistant and uniform with a stable spacing of $0.52 \mathrm{~ns}$. Correspondingly, the optical spectrum in linear scale is well fitted by Gaussian function, showing the feature of stretched pulses, as presented in Fig. 3(b). In particular, the emitting wavelength is 1594.97 $\mathrm{nm}$ with $10.19 \mathrm{~nm} 3 \mathrm{~dB}$ bandwidth locating at $\mathrm{L}$ band. It can be deduced that the red shift of the typical emitting wavelength of $1550 \mathrm{~nm}$ is attributed to intra-band absorption under the circumstance of the long gain medium. The typical autocorrelation trace is plotted in Fig. 3(c) from which we can see that it has a Gaussian-like profile which is in accordance with the shape of spectrum. The measured pulse duration is 742 fs, thus the time bandwidth product (TBP) is about 0.89, suggesting a slight pulse chirp. Additionally, the stability of the pulses is expressed by the recorded RF spectra as shown in Fig. 3(d), where the SMSR is $40.5 \mathrm{~dB}$ and the signal-to-noise ratio (SNR) is $56 \mathrm{~dB}$.

Under $233 \mathrm{~mW}$ pump power, the Q-switching operation emerges frequently indicating the multiphoton effect threshold of the CNT film. Thus we decrease pump power to $228 \mathrm{~mW}$,
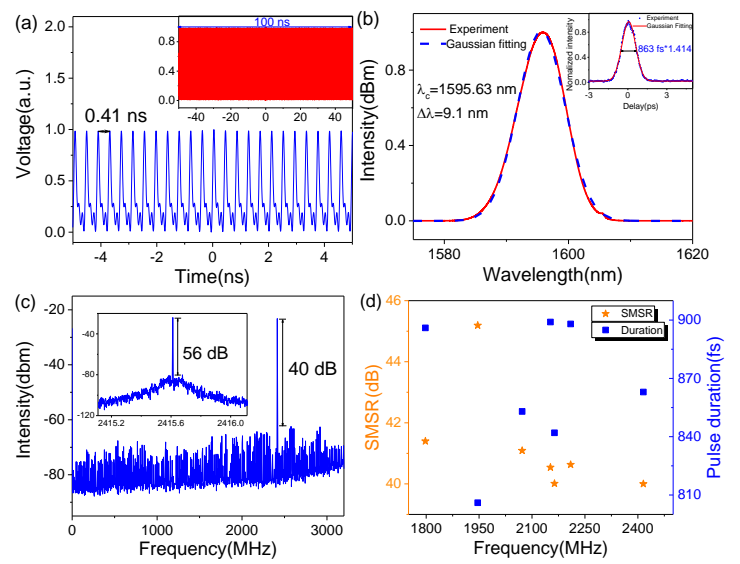

Fig. 4. (a) The oscilloscope trace with a repetition rate of $2.415 \mathrm{GHz}$. Inset: the pulse train spanning $100 \mathrm{~ns}$. (b) The corresponding optical spectrum in linear scale. Inset: autocorrelation trace fitted by Gaussian profile. (c) The RF spectra whose pronounced peak locates $2.415 \mathrm{GHz}$. (d) The variations of SMSR and pulse duration when pulses frequency ranges from $1.8 \mathrm{GHz}$ to $2.415 \mathrm{GHz}$.

and the pulses frequency ranges from $1.8 \mathrm{GHz}$ to $2.415 \mathrm{GHz}$ by adjusting the intracavity PC properly. Figure 4 shows the performances of $2.415 \mathrm{GHz}$ pulses corresponding to $213^{\text {rd }}$ harmonic of fundamental frequency. It is the highest record obtained in our experiment. The pulses uniformly distribute along the cavity with clearly smaller equal spacing ( $0.41 \mathrm{~ns})$ compared to Fig. 3(a), declaring higher frequency. This is the highest repetition rate achieved in CNTs-based PHML fiber laser working in L-band region. From Fig. 4(b), it is found that the profiles of the optical spectrum and autocorrelation trace are similar to those depicted in Fig. 3. And at this point, the $3 \mathrm{~dB}$ bandwidth is $9.1 \mathrm{~nm}$ and the pulse duration is $863 \mathrm{fs}$, resulting in TBP of 0.925 and DBP of 19.4. We should like to emphasize that the higher pulse frequency arises from the narrower spectral bandwidth resulting in smaller DBP value, which is coincident with Ref [12].Obviously, the outstanding stability is well maintained, which is examined by RF spectra as shown in Fig. 4(c), where the SMSR is $40 \mathrm{~dB}$ along with a $56 \mathrm{~dB}$ SNR. In addition, the output power is $5.83 \mathrm{~mW}$, indicating pulse energy of $2.41 \mathrm{pJ}$. Such low pulse energy indicates that the effective cavity management is successfully carried out. Worthy of mentioning, pulse duration maintains in the range of $800 \mathrm{fs}$ to $900 \mathrm{fs}$ and SMSR is always up to $40 \mathrm{~dB}$, as plotted in Fig. 4(d). It is postulated that the enhancement of pulse performances stems from the relatively broader spectral bandwidth and the reduction of dispersive wave when laser is organized in near-zero dispersion region [5][14].

Figure 5(a) summarizes pulses repetition rate and harmonic order in pace with pump power. As we can see, when increasing pump power from $161 \mathrm{~mW}$ to $233 \mathrm{~mW}$, the repetition rate increases from $629.65 \mathrm{MHz}$ to $1923.34 \mathrm{MHz}$ corresponding to harmonic order increase from $56^{\text {th }}$ to $170^{\text {th }}$. Both tendencies possess linear slope. Notably, the repetition rate slope is estimated to be $17.21 \mathrm{MHz} / \mathrm{mW}$, outperforming that of all previous reports. It may result from low pulse energy and low modulation depth of CNTs-SA [17], which will be further studied in our future work. Indeed, once HML has settled, it can be kept for several hours unveiling the long-term stability. It should be also noted that the optical spectrum is always in the 


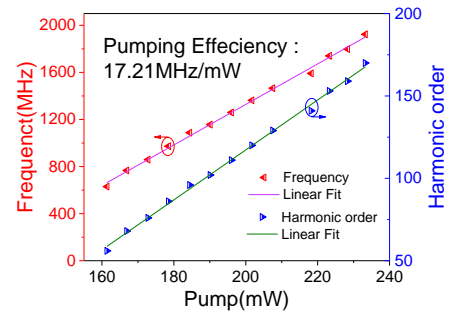

Fig. 5. (a) The laser frequency (red triangle) and harmonic order (blue triangle) as a function of pump power.

L band under all harmonic orders. Unfortunately, Q-switched pulses appear instead of higher repetition rate pulses when higher pump power is coupled into the cavity, resulting in high pulse energy, which may damage the CNTs SA film.

As far as we are concerned, there is no affirmatory mechanism implemented to clarify the formation of HML. Thereby, merely proceeding from our experimental phenomena, we postulate that HML originates possibly from the global interaction force mediated by $\mathrm{CW}$ component and the repulsive force induced by acousto-optic effect. Initially, pulses in a round trip move ceaselessly within the cavity with relatively random phase. Meanwhile, we find that $\mathrm{CW}$ co-exist with pulses in the corresponding optical spectrum and strong background noise appears in the time domain, suggesting the characteristic of unstable CW. Regarding the unstable CW, each pulse within the cavity is subjected to varying degrees of turbulence, resulting in different central frequency shift [15]. Therefore, the global interaction force is induced and plays a major role in the movement of pulses [18]. Afterwards, if we adjust $\mathrm{PC}$ carefully to suppress the unstable $\mathrm{CW}$ component, the pulse sequence is then spaced equally in a short time. Enlightened by analysis expressed in Ref [19], we conjecture that the self-stabilized behavior comes from the pulse-to-pulse repulsive force induced by acousto-optic effect when the global interaction induced by $\mathrm{CW}$ is weak.

\section{CONCLUSION}

In conclusion, we have demonstrated a high-order PHML Erdoped fiber laser based on SWCNTs-PVA film working in L band. When pump laser delivers up to $233 \mathrm{~mW}$ pump light, $1.923 \mathrm{GHz}$ pulse train is realized with $40.5 \mathrm{~dB}$ SMSR at $170^{\text {th }}$ harmonic. Furthermore, at pump power of $228 \mathrm{~mW}$, the laser enables $1.8 \mathrm{GHz}$ to $2.415 \mathrm{GHz}$ stable pulses generation when we adjust PC appropriately. The pulse duration varies from 800 fs to $900 \mathrm{fs}$ while the SMSR constantly maintained over $40 \mathrm{~dB}$. In comparison with the pulse performances reported in Ref [12], it possesses more advantageous pulse properties, particularly the pulse duration and stability. Specifically, the highest repetition rate pulse delivered from the laser is $2.415 \mathrm{GHz}$ corresponding to $213^{\text {rd }}$ harmonic, which, to the best of our knowledge, is a record high levels obtained from a L-band PHML laser using CNTs. We believe that such high repetition rate short duration pulses combining outstanding stability together with L-band emission show the superiority for some specific applications such as modern optical communication system. Also, it is worth noting that the pump harmonic efficiency considerably enhances to $17.21 \mathrm{MHz} / \mathrm{mW}$, compared to that in other reports. Additionally, from the experimental observations, we have proffered a qualitative explanation for the formation of HML, where the CW component and acoustooptic effect play a great role. However, the more plausible and quantitative explanation calls for further simulation and experiment. Besides, the time jitter and relative intensity noise should be measured in our future work.

\section{REFERENCES}

[1] R. J. Jones and J.-C. Diels, "Stabilization of femtosecond lasers for optical frequency metrology and direct optical to radio frequency synthesis," Phys. Rev. Lett., vol. 86, no. 15, pp. 3288, Apr. 2001.

[2] J. B. Schlager, P. D. Hale, and D. L. Franzen, "High-sensitivity optical sampling using an erbium-doped fiber laser strobe," Microwave Opt. Technol. Lett., vol. 6, no. 15, pp. 835-837, Dec.1993.

[3] H. A. Haus and W. S. Wong, "Solitons in optical communications," Rev. Mod. Phys., vol. 68, no. 2, pp. 423-444, Apr. 1996.

[4] W.-W. Hsiang, C.-Y. Lin, M.-F. Tien, and Y. Lai, "Direct generation of a $10 \mathrm{GHz} 816$ fs pulse train from an erbium-fiber soliton laser with asynchronous phase modulation," Opt. Lett., vol. 30, no. 18, pp. 2493-2495, Sep. 2005.

[5] Y. Wang, H. Tian, Y. Ma, Y. Song, and Z. Zhang, "Timing jitter of highrepetition-rate mode-locked fiber lasers," Opt. Lett., vol. 43, no. 18, pp. 4382-4385, Sep. 2018.

[6] G. Sobon, J. Sotor, and K. M. Abramski, "Passive harmonic mode-locking in Er-doped fiber laser based on graphene saturable absorber with repetition rates scalable to $2.22 \mathrm{GHz}, "$ Appl. Phys. Lett., vol. 100, no. 16, pp. 161109-1-161109-4, Apr. 2012.

[7] A. Grudinin, D. Richardson, and D. Payne, "Passive harmonic modelocking of a fibre soliton ring laser," Electron. Lett., vol. 29, no. 21, pp. 1860-1861, Oct. 1993.

[8] Y. Deng, M. Koch, F. Lu, G. W. Wicks, and W. H. Knox, "Colliding-pulse passive harmonic mode-locking in a femtosecond $\mathrm{Yb}$-doped fiber laser with a semiconductor saturable absorber," Opt. Express, vol. 12, no. 16, pp. 3872-3877, Aug. 2004.

[9] K. Jiang, S. Fu, P. Shum, and C. Lin, "A wavelength-switchable passively harmonically mode-locked fiber laser with low pumping threshold using single-walled carbon nanotubes," IEEE Photonics Technol. Lett., vol. 22, no. 11, pp. 754-756, Jun. 2010.

[10] C. Mou, R. Arif, A. Rozhin, and S. Turitsyn, "Passively harmonic mode locked erbium doped fiber soliton laser with carbon nanotubes based saturable absorber," Opt. Mater. Express, vol. 2, no. 6, pp. 884-890, May. 2012.

[11] C. S. Jun, S. Y. Choi, F. Rotermund, B. Y. Kim, and D.-I. Yeom, "Toward higher-order passive harmonic mode-locking of a soliton fiber laser," Opt. Lett., vol. 37, no. 11, pp. 1862-1864, Jun. 2012.

[12] Q. Huang, C. Zou, T. Wang, M. Al Araimi, A. Rozhin, and C. Mou, "Influence of average cavity dispersion and spectral bandwidth on passively harmonic mode locked L-band Er-doped fiber laser," IEEE JSTQE, vol. 25, no. 4, pp. 1-8, July-Aug. 2019.

[13] H. Kataura et al., "Optical properties of single-wall carbon nanotubes," Synth. Met., vol. 103, no. 1-3, pp. 2555-2558, Jun. 1999.

[14] S. Kelly, "Characteristic sideband instability of periodically amplified average soliton," Electronics Letters, vol. 28, no. 8, pp. 806-807, Apr. 1992.

[15] D. Y. Tang, B. Zhao, L. M. Zhao, and H. Y. Tam, "Soliton interaction in a fiber ring laser," Phys. Rev. E: Stat. Nonlinear Soft Matter Phys., vol. 72, no. 2, pp. 016616-1-016616-10, July. 2005.

[16]Z. Zhang, L. Zhan, X. Yang, S. Luo, and Y. Xia, "Passive harmonically mode-locked erbium-doped fiber laser with scalable repetition rate up to 1.2 GHz," Laser Phys. Lett., vol. 4, no. 8, pp. 592-596, Feb. 2007.

[17] J. Bogusławski, G. Soboń, R. Zybała, and J. Sotor, "Towards an optimum saturable absorber for the multi-gigahertz harmonic mode locking of fiber lasers," Photonics Res., vol. 7, no. 9, pp. 1094-1100, Sep. 2019.

[18] B. Zhao et al., "Passive harmonic mode locking of twin-pulse solitons in an erbium-doped fiber ring laser," Opt. Commun., vol. 229, no. 1, pp. 363370, Jan. 2004.

[19]A. B. Grudinin and S. Gray, "Passive harmonic mode locking in soliton fiber lasers," J. Opt. Soc. Am. B: Opt. Phys., vol. 14, no. 1, pp. 144-154, Jun. 1997. 